\title{
LOCAL COMPLICATIONS OCCURRING DURING DENTAL IMPLANTATION
}

\author{
Georgiev T.*, Nogalchev K. \\ *Department of Oral and Maxillofacial Surgery, \\ Department of Prosthetic Dental Medicine, \\ Faculty of Dental Medicine, Medical University - Varna, Bulgaria
}

\begin{abstract}
With regard to the emergence of new concepts in dental treatment involving placement of dental implants and the significance of therapeutic treatment of the intrusion in their complications. The purpose of the article is to make a review of the problems and to point out options for their treatment.
\end{abstract}

Key words: periimplantitis, dental implants, complications, failures

The classical subdivision of complications into iatrogenic factors, biological and mechanical, does not fully reflect their nature. For example, the complications considered as biologically caused during the stage of healing with formation of connective tissue, may be caused due to overheating during the preparation of the implant bed with tissue necrosis, which is included in the iatrogenic factors.

In relation to that, the classification has to be made in view of the different stages, i.e. to review the complications in chronological order depending on the stages of implantation.

Complications that occur during:

the stage of the initial intrusion;

at the time of healing;

at the time of uncovering the implant;

the performance of orthopedic actions.

During the stage of initial intrusion, the complications are mainly manifested as bleeding, perforation of the osseous structure and the external cortical plate of the lower jaw, as well as damage of the mandibular nerve.

\section{BLEEDING}

In the literature there are described 5 cases of dangerous bleedings, occurring at the stage of surgical insertion of the implant. Similar complications occur when the compact layer of the bone is perforated. The literature describes cases in which a branch of a.lingualis is damaged, involving bleeding in the region of the floor of the oral cavity, leading to a large hematoma and disorder of the respiratory function.
In similar situations, the hematoma has to be removed and subsequent intensive treatment is to be carried out.

\section{Perforation of the sinus cavity}

In case of incorrect judgment with regard to the distance which we have available between the alveolar ridge and the bottom of the sinus cavity, the latter is often perforated. If the implant is movable, or is located in the sinus cavity, it has to be removed, subsequently the sinus cavity has to be closed using the methods of guided tissue regeneration. Perforation in case of initial stability of the implant and intact mucosa of the sinus cavity does not have a negative effect on the implant prognosis; to the contrary, recently many sources state that the bicortical fixation of the implant gives it additional initial stability and the chance to stand higher chewing forces.

In case of laceration of the sinus mucosa, it is possible to be caused bleeding from the nose and a hematoma on the respective side of the maxillary sinus. If bleeding can be stopped and there is enough space for the initial fixation of the implant, it is not necessary to delay the implantation itself.

\section{Damaging $n$. alveolaris inferior}

One of the most severe local complications is the damage of the osseous roof of the mandibular canal and the soft tissues in it, which is caused by incorrect planning of implantation, incorrect reading of the X-ray or tomography, or lack of good enough surgical habits of the dental doctor which will allow them to put into practice the correct planning.

Seddon has described 3 types of nerve damage:

Neuropraxia - temporary impairment of sensitivity, without anatomic damage of the bone, sensitivity is restored spontaneously after about 4 weeks. In dental implantation it may occur when the generally accepted limit of $2 \mathrm{~mm}$ gap between the dental implant and the mandibular canal is exceeded, thereby causing pressure on $\mathrm{n}$. alveolaris inferior, which may lead to this condition;

Axonotmesis - this is a more serious condition in which there is partial anatomic damage of the continuity of 
the nerve and partial degeneration of the nerve which is located distally to the damage. The initial symptoms of recovery will not occur earlier than 6-8 weeks after the trauma. Recovery may be incomplete (hypoesthesia) and is often accompanied by sensations of pain (dysesthesia). It may occur in dental implantation when the implant partially tears $n$. alveolaris inferior;

Neurotmesis - this is complete interruption of the nerve continuity with total degeneration of the section which lies distally to the damage. The patient feels complete anesthesia at least for 3 months and there is a probability for this state to be wholly or partially preserved forever.

A similar complication is also the damage of n.lingualis caused by rotating tools during the preparation of bone cavity, or due to incorrect attitude and work with the soft tissues in the distal sections (lingually) of the lower jaw. Consequences are identical to those of the damage of n. alveolaris inferior.

\section{Exposure of the parts of the implant}

Incorrect placing of the implant in the buccal or lingual aspects or the different anatomic conditions cause exposure of the implant neck and a part of its body. Similar conditions are often met in practice and are solved using the methods of osseous augmentation.

\section{Complication during the healing of implant}

These complications may be divided into soft or hard tissue connected complications.

The complications connected to soft tissues are:

- exposure of the implant;

- formation of a fistula;

- opening of the edges of the wound.

Perforation of the mucosa most often results from mechanical irritation by the implant due to insufficient depth of insertion. As a possible reason may also be considered the prosthesis, placed above the implant, which is not adequately adapted, lying closely to the mucosa above the implant and resulting in its exposure due to mechanical irritation.

If perforation of the implant mucosa is ascertained several weeks after implantation, the ] treatment is not mandatory in case of satisfactory hygienic measures carried out by the patient. In case of poor hygiene, signs of local inflammation or unfavorable aesthetic situation, perforation has to be plastically closed, after preliminary refreshment of the wound edges and removal of granulation tissue, if any.

The main reason for the occurrence of fistulae of the soft tissues around dental implants is the mobility of the cover screw, the residues of suturing material or change in the gums. Treatment consists of tighter screwing of the cover screw, removal of the foreign material and circlewise cutting of the changed gingival tissues.

Very rarely the fistula is due to inadequately cleaned alveolus from granulation tissue in the region of the apex of the extracted tooth, with subsequent single-moment insertion of an implant. Treatment is the same as in apical osteotomy of native teeth - exact cleaning of the field from granulations, refreshment of the wound edges, cutting of the fistular course and filling in the defect with osteoplastic materials.

The complications related to the hard tissues mainly include the mobility of the implant which is determined by an X-ray as periimplant osteolysis. The loose implant is evaluated as lack of osseous integration and it has to be extracted.

It is possible that periimplantitis occurs without a loose or mobile implant (i..e loss of bone with exposure of a part of the implant body). In this case the inflamed focus has to be cleaned and guided bone regeneration to be made in order to restore the osseous tissues around the implant.

\section{Complications occurring at the stage of uncovering the implant}

- bone cover of the screw;

- incorrect placement of the abutment;

- too short or too long abutment.

The covering of the cover screw by osseous tissue is most often caused by inserting the implant too deeply in the bone. In this case it is recommended to remove ], because of the inability to place the abutment.

Short or too long superstructure. Short superstructures do not ensure good attachment to the bridge structures which will be used after that. They are convenient only if screwed-in structures are to be used. Superstructures which are tool long necessitate for them to be adjusted in the laboratory which entails loss of time and resources.

Superstructures should have an angle suitable for subsequent restoration with bridge structures, otherwise their retention is corrupted, leading to a number of complications - compromising of the already integrated implant, soft tissue traumas.

\section{Complications occurring during the stage of preparation of implant suprastructure}

Complications from overloading of the implant most often these are fractures of:

- the level of the superstructure - fracture of the superstructure due to inadequate planning of the prosthetic treatment, usually the fracture is in the transverse screw which attaches the superstructure to the implant. If the screw can be unscrewed, new prosthetic planning and treatment have to be undertaken. If the crew cannot be unscrewed, the implant has to be removed and a new one to be placed.

- fracture of the implant - Fracture of the implant neck - occurs when the implant is overloaded, in case of improper choice of superstructures, poor planning of structures, poor occlusal and articulation proportions. If it 
cannot be built with the use of methods of prosthetic dentistry, the implant has to be substituted.

- fracture of the implant body - it occurs when the implant is overloaded, it may not be due to the planned treatment, in people with parafunctions such as bruxism and bruxomania who do not use occlusal splints. Treatment involves change of implant or new planning of the whole surgical and prosthetic treatment.

The progressive destruction of the marginal sections of osseous tissue may be caused by inflammation of the surrounding tissues adjacent to the implants, inadequate loading of the implant or a mistake in making the suprastructure.

The above complications may be avoided by means of careful planning of implantation and its competent implementation at each stage of intrusion. Additional control tests of the patients will allow to promptly detect and eliminate the mistakes and the causes which lead to them.

\section{REFERENCES:}

1. Anzalone JV, Vastardis S. Oroantral communication as an osteotome sinus elevation complication. J Oral Implantol. 2010; 36(3):231-7.

2. Gealh W, Mazzo V, Barbi F, Camarini E. OSSEOINTEGRATED IMPLANTS FRACTURE: CAUSES AND TREATMENT. J Oral Implantol. 2010 Jun 16.

3. Brдgger U, Karoussis I, Persson R, Pjetursson B, Salvi G, Lang N. Technical and biological complications/failures with single crowns and fixed partial dentures on implants: a 10-year prospective cohort study. Clin Oral Implants Res. 2005 Jun; 16(3):326-34.
4. Schug T, Dumbach J, Rodemer H. [Mandibular fracture. An unusual implantation complication] Mund Kiefer Gesichtschir. 1999 Nov; 3(6):335-7. German.

5. Fujioka M, Oka K, Kitamura R, Yakabe A, Endoh H. Extra-oral fistula caused by a dental implant. J Oral Implantol. 2010 Jun 14

6. Tord Berglundh, Leif Persson, Bjorn Klinge, A systematic review of the incidence of biological and technical complications in implant dentistry reported in prospective longitudinal studies of at least 5 years; Journal of Clinical
Periodontology Volume 29 Issue s3, Pages 197 - 212, Published Online: 15 May 2003

7. Charles J. Goodacre, Joseph Y.K. Kan, Kitichai Rungcharassaeng, Clinical complications of osseointegrated implants. Journal of prosthetic dentistry, Volume 81, Issue 5, Pages 537-552 (May 1999)

8. Charles J Goodacre, Guillermo Bernal, Kitichai Rungcharassaeng, Joseph Y. K Kan. Clinical complications with implants and implant prostheses Journal of prosthetic dentistry, Volume 90, Issue 2, Pages 121-132 (August 2003)

\section{Correspondence address:}

Dr Tihomir Georgiev

18 Hristo Popovich St., Varna 9000, Bulgaria

e-mail: tgeorgeivphd@yahoo.com 